\title{
Comparative analysis of efficacy and tolerability of tramadol versus diclofenac in treatment of knee osteoarthritis
}

\author{
Rima Kanjiya, Ravi Kotadia*
}

Department of Pharmacology, PDU government medical college Rajkot, Gujarat, India

\author{
Received: 05 August 2021 \\ Revised: 01 September 2021 \\ Accepted: 02 September 2021 \\ *Correspondence: \\ Dr. Ravi Kotadia, \\ Email: drravikotadia44@gmail.com
}

Copyright: $\odot$ the author(s), publisher and licensee Medip Academy. This is an open-access article distributed under the terms of the Creative Commons Attribution Non-Commercial License, which permits unrestricted non-commercial use, distribution, and reproduction in any medium, provided the original work is properly cited.

\begin{abstract}
Background: Present study was conducted on patients of osteoarthritis (OA) treated with the traditional drug diclofenac sodium and compares it with tramadol, with the primary objective for determining effectiveness and tolerability based on WOMAC scores.

Methods: The study was conducted as a randomized clinical study of the effectiveness and tolerability of tramadol versus diclofenac in the treatment of knee OA, at Tertiary care institute of Gujarat in the department of orthopedics. Consecutive patients were allotted serially in two groups; tramadol group $(n=75)$ One tablet orally twice daily for 8 weeks. Diclofenac group (n75) patients received $75 \mathrm{mg}$ oral dose of diclofenac 2 times daily for a period of 8 weeks. Overall improvement was evaluated through various parameters.

Results: About $33 \%$ decrease in the scoring of stiffness domain of the WOMAC OA index questionnaire in the tramadol group and a $21 \%$ decline in the diclofenac group was observed. There was a $65 \%$ decrease in the scoring of knee joint tenderness questionnaire in the tramadol group and a $44 \%$ reduction in the diclofenac group. Tramadol was found to be more effective in improving the WOMAC OA index. WOMAC score (overall) and knee joint tenderness scores were also improved by tramadol than diclofenac.

Conclusions: There was a significant improvement in the standard of life in patients suffering from knee OA when treated with tramadol. Tolerability was better with tramadol than diclofenac.
\end{abstract}

Keywords: Diclofenac, Knee joint, Osteoarthritis, Tramadol

\section{INTRODUCTION}

Osteoarthritis (OA) is a chronic, inflammatory joint disease in the whole world. ${ }^{1}$ In India, more than $20 \%$ of the total population is suffering from arthritis; although the chief cause of the disease is unknown, morphological changes witnessed in OA include cartilage erosion as well as inflammation. ${ }^{2}$ The most affected joints include the hips, knees, back and neck, joints of the fingers, the base of the thumb, and big toe. ${ }^{3} \mathrm{OA}$ is more common in women than in men. The worldwide estimates are that $9.6 \%$ of men and $18 \%$ of women aged more than 60 years have symptomatic OA. ${ }^{4}$
Risk factors for OA include advanced age, female gender, genetic predisposition, obesity, and joint injury including trauma, repetitive use, and prior inflammation. ${ }^{5-8}$ Genes that encode collagen type II have been proposed as candidate genes for familial OA. ${ }^{9,}{ }^{10}$ Radiographs can help confirm OA when the diagnosis is uncertain from clinical examination. It is usually not difficult to differentiate OA from a systemic rheumatic disease, such as rheumatoid arthritis, because joint involvement in the latter disease is usually symmetric and polyarticular, with arthritis in wrists and metatarsophalangeal joints and constitutional features such as prolonged morning stiffness, fatigue, weight loss, or fever may be seen. ${ }^{11}$ Synovial fluid analysis 
reveals mild leukocytosis is i.e., with a predominance of mononuclear cells. Synovial fluid analysis is of particular value in excluding other conditions, such as calcium pyrophosphate dehydrates deposition disease, gout or septic arthritis. ${ }^{12}$

The main oral pharmacological options currently used to treat pain caused by OA include paracetamol, nonsteroidal anti-inflammatory drugs (NSAIDs) and opiates. ${ }^{13}$ Recently, the international osteoarthritis research society (OARSI) has published a series of recommendations based on review of available guidelines for the management of patients with OA of the hip and knees. ${ }^{14}$ They recommend taking the lowest effective dose of NSAIDs, avoiding long-term use, as they are associated with dose and duration-related risks of gastrointestinal, cardiovascular, and renal-function adverse events (AEs). They also recommend the use of a gastro protective agent, such as a proton pump inhibitor (PPI), with oral NSAIDs to reduce gastrointestinal adverse events (AEs). Diclofenac is a nonselective COX inhibitor type belonging to the heterocyclic arylacetic acid derivative subtype of nonsteroidal anti-inflammatory drugs. Conventionally, diclofenac sodium has been used in the treatment of OA. However, diclofenac has shown an increase in the incidence of adverse effects such as abdominal pain, dyspepsia, diarrhea, heartburn, and GI ulcer. Therefore, tramadol is now being increasingly used in OA. Tramadol is a centrally acting analgesic with the opioid-like activity of tramadol derives from low-affinity binding to $\mu$-opioid receptors and higher affinity binding of the principal active metabolite, O-desmethyl tramadol, denoted M1, to $\mu$ opioid receptors, and it is a commonly used opioid. ${ }^{15}$ Tramadol has a better ability to reduce pain which is primarily responsible for limitation and disability in patients with OA. Greater reduction in pain leads to a better quality of life in patients with OA. There is a paucity of data where a comparison between these two drugs has been done in the Indian population. Hence, we conducted a study on patients of OA treated with the traditional drug diclofenac sodium and compare it with tramadol, with the primary objective for determining effectiveness and tolerability based on WOMAC scores.

\section{METHODS}

The study was conducted as a randomized clinical study of the effectiveness and tolerability of tramadol versus diclofenac in the treatment of knee OA, at Tertiary care institute of Gujarat in the department of orthopedics from January 2020 to August 2020. Ethical approval was taken from the institutional ethical committee and written informed consent was taken from all the participants.

\section{Inclusion criteria}

Male and female patients who were $\geq 39$ years of age and radiological diagnosed with osteoarthritis of the knee were included in the study.

\section{Exclusion criteria}

Patients with a history or showing the presence of other rheumatic disease that would be responsible for secondary osteoarthritis, patients with a history of peptic ulcers, patient with a history of bleeding disorders, patients with renal impairment, alcoholic liver disease, pregnant or lactating woman, uncontrolled medical conditions like severe anemia, hypertension, congestive cardiac failure and bronchial asthma were excluded from the study.

Consecutive patients were allotted serially in two groups; tramadol group $(n=75)$ One tablet orally twice daily for 8 weeks. Diclofenac group $(n=75)$ patients received $75 \mathrm{mg}$ oral dose of diclofenac 2 times daily for a period of 8 weeks. The patients are selected from an age group since this is age group commonly involved with OA.

\section{Follow up}

After 2 months of taking of treatment patients were followed up by following methods: The change in the scoring of pain domain of WOMAC OA index, the change in the scoring of stiffness domain of WOMAC OA index, the change in the scoring of difficulty in performing daily activities (DPDA) domain of WOMAC OA index, the change in WOMAC score (overall), the change in the scoring of knee joint tenderness, tolerability was assessed based on the adverse events reported.

\section{Statistical analysis}

The recorded data was compiled and entered in a spreadsheet computer program (Microsoft excel 2007) and then exported to data editor page of SPSS version 15 (SPSS Inc., Chicago, Illinois, USA). For all tests, confidence level and level of significance were set at $95 \%$ and $5 \%$ respectively.

\section{RESULTS}

Among the total $n=150$ patients, $n=90$ patients were females and $n=60$ were males. In the diclofenac group, there were $n=46$ females and $n=29$ males and in the tramadol group, there were $n=29$ females and $n=31$ males. The change in the scoring of stiffness domain of the WOMAC OA index questionnaire in both the study groups was measured (Table 1). About 33\% decrease in the scoring of stiffness domain of the WOMAC OA index questionnaire in the tramadol group and a $21 \%$ decline in the diclofenac group was observed. The change in the scoring of DPDA domain of the WOMAC OA index in both the study groups was assessed. A $27 \%$ decrease in the scoring of DPDA domain of WOMAC OA index questionnaire scoring in the tramadol group and a $22 \%$ reduction in the diclofenac group were observed (Table 1). The change in the WOMAC score (overall) in both the study groups was analyzed. A $32 \%$ decrease in the WOMAC score (overall) in the tramadol group and a $23 \%$ decline in the diclofenac group were seen. 
The change in the scoring of knee joint tenderness in both the study groups was assessed (Table 2). There was a $65 \%$ decrease in the scoring of knee joint tenderness questionnaire in the tramadol group and a $44 \%$ reduction in the diclofenac group. Tramadol was found to be more effective in improving the WOMAC OA index. WOMAC score (overall) and knee joint tenderness scores were also improved by tramadol than diclofenac. Diclofenac and tramadol groups were compared by paired t-test and unpaired t-test, they were found to be statistically significant in parameters in study groups. When the mean difference within the two study groups was compared statistically, the change in the scoring of pain domain of the WOMAC OA index was $\mathrm{p}<0.05$. The mean difference within the two study groups was compared and the change in the scoring of stiffness domain of WOMAC OA index was $\mathrm{p}<0.05$. The comparative analysis of the mean difference in the two study groups was statistically analyzed; the change in the scoring of DPDA domain of the WOMAC OA index was $\mathrm{p}<0.05$. The mean difference within the two study groups was compared by t-test, the change in WOMAC score (overall) was $p<0.05$. Mean difference within the tramadol and diclofenac groups was statistically compared, the change in the scoring of knee joint tenderness was $\mathrm{p}<0.05$. In the tramadol group, one patient reported epigastric discomfort, two patients complained of dyspepsia, one patient reported diarrhea, two patients complained of nausea, three patients suffered constipation, and two patients complained of flatulence. Within the diclofenac group, five had epigastric discomfort, four complained of dyspepsia, two had diarrhea, four had nauseas, two had constipation, and one complained of flatulence.

Table 1: The change in the scoring of pain domain, stiffness domain, and DPDA domain of the WOMAC osteoarthritis index and WOMAC score (overall) in both the study groups.

\begin{tabular}{|lllllll|}
\hline & \multicolumn{2}{c}{ Tramadol group } & \multicolumn{3}{c|}{ Diclofenac group } \\
\hline Variable & $\mathbf{1}^{\text {st }}$ visit & $\mathbf{2}^{\text {nd }}$ visit & P value & $\mathbf{1}^{\text {st }}$ visit & $\mathbf{2}^{\text {nd }}$ visit & P value \\
\hline $\begin{array}{l}\text { Scoring of pain domain } \\
\text { questionnaire }\end{array}$ & $11.90 \pm 1.21$ & $7.10 \pm 2.02$ & $0.05^{*}$ & $11.85 \pm 1.26$ & $8.2 \pm 1.11$ & $0.004^{*}$ \\
$\begin{array}{l}\text { Scoring of stiffness domain } \\
\text { questionnaire }\end{array}$ & $4.03 \pm 0.3$ & $2.40 \pm 0.6$ & $0.001 *$ & $4.2 \pm 0.4$ & $3.1 \pm 0.2$ & $0.03 *$ \\
\hline $\begin{array}{l}\text { Scoring of DPDA domain } \\
\text { questionnaire }\end{array}$ & $39.9 \pm 2.5$ & $29.1 \pm 02.01$ & $0.02 *$ & $39.1 \pm 2.7$ & $30.01 \pm 1.22$ & $0.001 *$ \\
\hline WOMAC score (overall) & $55.9 \pm 2.4$ & $38.8 \pm 2.12$ & $0.01 *$ & $56.2 \pm 3.40$ & $41.9 \pm 01.1$ & $0.02 *$ \\
\hline *Indicates statistically significance at $\mathrm{p} \leq 0.05$ & & & & &
\end{tabular}

Table 2: Change in the scoring of knee joint tenderness.

\begin{tabular}{|lllllll|}
\hline & \multicolumn{2}{c|}{ Tramadol group } & \multicolumn{3}{c|}{ Diclofenac group } \\
\hline Variable & $\mathbf{1}^{\text {st }}$ visit & $\mathbf{2}^{\text {nd }}$ visit & P value & $\mathbf{1}^{\text {st }}$ visit & $\mathbf{2}^{\text {nd }}$ visit & P value \\
\hline Scoring of knee joint tenderness & $1.31 \pm 0.22$ & $0.41 \pm 0.28$ & $0.01^{*}$ & $1.11 \pm 0.97$ & $0.80 \pm 0.4$ & $0.05^{*}$ \\
\hline *Indicates statistically significance at $\mathrm{p}<0.05$ & & & &
\end{tabular}

\section{DISCUSSION}

Until recently the new COX-2 selective inhibitors have been increasingly used. They have equal efficacy to standard NSAIDs. ${ }^{16,17}$ In the study, there has been a decrease in all three domains of the WOMAC OA index questionnaires scoring with both the tramadol group and the diclofenac group. In this study, there was a $15 \%$ decrease in time taken to walk 100 feet in the diclofenac group and a $17 \%$ decrease in the tramadol group. We found that there was a $41 \%$ decrease in pain score at rest in diclofenac and a $49 \%$ decrease in the tramadol group. A decrease in pain score during active movement was $32.4 \%$ in diclofenac and $44.8 \%$ decrease in the tramadol group. The results also revealed that there was a $44 \%$ decrease in joint tenderness score in diclofenac and $65 \%$ of the tramadol group which was significant. Tramadol was superior to diclofenac in decreasing joint tenderness score clinically as well as statistically. Assessment of disease status and response to therapy was made on a 0-4 Likert scale by the investigators. There was a $44 \%$ improvement in disease status in the diclofenac group and a $65 \%$ increase in the tramadol group. Assessment of response to the drug was made on a 0-4 Likert scale by the patient. Patient response to tramadol was better than diclofenac clinically as was as statistically. Pareek et al ${ }^{18}$ compared aceclofenac with diclofenac and it was found that aceclofenac was superior to diclofenac in decreasing WOMAC score. Ward et al $^{19}$ found aceclofenac and diclofenac were equally effective in decreasing pain on weight-bearing, pain at rest, and pain during active movements on VAS. Sridhar et al comparing the efficacy of tramadol and aceclofenac in the treatment of OA found that aceclofenac was efficacious as compared to tramadol in the treatment of OA. ${ }^{20}$ Malonne et al studying the tolerability of tramadol in the treatment of OA found that long-term treatment with tramadol once daily was generally safe in cases of OA. ${ }^{21}$ Tramadol is an analgesic that has incredible use in acute pain, chronic pain, cancer pain, etc., mostly due to its dual mode of action. ${ }^{22}$ In an earlier study, IR tramadol was compared with IR 
diclofenac in OA patients. ${ }^{23}$ Both medications were given as needed, to a maximum dose of $300 \mathrm{mg}$ /day for tramadol and $150 \mathrm{mg} /$ day for diclofenac. Correspondingly, patients experienced greater functional improvement in the overall WOMAC pain, stiffness and function scores in the present study, compared with the study. ${ }^{23}$ But as evident from the graphs mean scores for all the parameters has substantially increased in all the treatment groups with maximum increase in DIC+PPI group reflecting its maximum efficacy compared to tramadol group. This can be because diclofenac gets distributed in synovial fluid and has chondro protective and other action as mentioned like blockage of voltage-dependent sodium channels and acidsensing ion channels (ASICs), positive allosteric modulation of KCNQ-and BK-potassium channels. ${ }^{24}$ And addition of PPI may be responsible for increase efficacy due to its anti-inflammatory action as well as antisecretory action which decreases gastrointestinal adverse effect and increases compliance..$^{25,26}$

Tramadol is an analgesic that has incredible use in acute pain, chronic pain, cancer pain, etc., mostly due to its dual mode of action. ${ }^{27}$ The effectiveness and safety of tramadol for musculoskeletal pain attributed to $\mathrm{OA}$ are spectacular. ${ }^{28}$ Some of the concerns with nonsteroidal antiinflammatory drugs (NSAIDs) are they are toxic to articular cartilage and have deleterious effects on bone healing. ${ }^{29,30}$ Although tramadol does not have antiinflammatory properties its central action could be of benefit as it decreases the central neuronal sensitization due to persistent nociceptive inputs. ${ }^{31}$ The incidences of ADRs are lesser with tramadol and it was better tolerated with fewer incidences of GI adverse effects as commonly seen with NSAIDs like an observation of other studies. ${ }^{32}$,

\section{CONCLUSION}

Since long term NSAID treatment is indicated for osteoarthritis, the ideal agent should have good efficacy and a low propensity to cause adverse events. There was a significant improvement in the standard of life in patients suffering from knee OA when treated with tramadol. Tolerability was better with tramadol than diclofenac. Although, clinicians may be compelled in some circumstances to use diclofenac due to its antiinflammatory properties which are required in cases of OA where inflammation is severe. Therefore, the use of clinical judgment is paramount in deciding the drugs to be used, and sometimes a combination of both can be tried under appropriate circumstances.

\section{Funding: No funding sources}

Conflict of interest: None declared

Ethical approval: The study was approved by the Institutional Ethics Committee

\section{REFERENCES}

1. Bortoluzzi A, Furini F, Scirè CA. Osteoarthritis and its management-epidemiology, nutritional aspects and environmental factors. Autoimmun Rev. 2018;17:1097-104.

2. Ashkavand Z, Malekinejad H, Vishwanath BS. The pathophysiology of osteoarthritis. J Pharm Res. 2013;7:132-8.

3. Engler D, Grootboom W, Mogale K. Osteoarthritis. SA Pharm J. 2018;85:43-50.

4. March L, Cross M, Arden NK. Osteoarthritis: A Serious Disease, Submitted to the U. S. Food and Drug Administration, OARSI; 2016. p. 1-103. Available at: https://www.oarsi.org/ sites/default/files/docs/2016/oarsi_white_paper_oa_s erious_disease_121416_1.pdf. Accessed on 2021 June 12.

5. Hagen ND, Olson T, Millett P. Comprehensive postarthroscopic management of a middle-aged adult with glenohumeral osteoarthritis: a case report. Int J Sports Phys Ther. 2013;8(1):54-61.

6. Macrini TE, Coan HB, Levine SM, Lerma $T$. Reproductive status and sex show strong effects on knee OA in a baboon model. Osteoarthritis Cartilage. 2013.

7. Haseeb A, Haqqi TM. Immunopathogenesis of osteoarthritis. Clin Immunol. 2013;146(3):185-96.

8. Koonce RC, Bravman JT. Obesity and osteoarthritis: more than just wear and tear. J Am Acad Orthop Surg. 2013;21(3):161-9.

9. Seki K, Fujimori T, Savagner P. Mouse Snail family transcription repressors regulate chondrocyte, extracellular matrix, type II collagen, and aggrecan. J Biol Chem. 2003;278(43):41862-70.

10. Li G, Han N, Li Z, Lu Q. Identification of transcription regulatory relationships in rheumatoid arthritis and osteoarthritis. Clin Rheumatol. 2013;32(5):609-15.

11. Duncan AE, Colman RJ, Kramer PA. Longitudinal study of radiographic spinal osteoarthritis in a macaque model. J Orthop Res. 2011;29(8):1152-60.

12. Liao W, Li Z, Wang H, Wang J. Proteomic analysis of synovial fluid: insight into the pathogenesis of knee osteoarthritis. Int Orthop. 2013;37(6):1045-53.

13. Barron MC, Rubin BR. Managing osteoarthritic knee pain. J Am Osteopath Assoc. 2007;10710(6):ES21-7.

14. Zhang W, Moskowitz RW, Nuki G, Abramson S, Altman RD, Arden N, et al. OARSI recommendations for the management of hip and knee osteoarthritis, Part II: OARSI evidence-based, expert consensus guidelines. Osteoar Thr Cartil. 2008;16:137-62.

15. Hooper L, Brown TJ, Elliott R, Payne K, Roberts C, Symmons D. The effectiveness of five strategies for the prevention of gastrointestinal toxicity induced by non-steroidal anti-inflammatory drugs: systematic review. BMJ. 2004;329:948.

16. Bäck M, Yin L, Ingelsson E. Cyclooxygenase-2 inhibitors and cardiovascular risk in a nation-wide cohort study after the withdrawal of rofecoxib. Eur Heart J. 2012;33(15):1928-33.

17. Carman WJ, Su S, Cook SF. coronary heart disease outcomes among chronic opioid and cyclooxygenase- 
2 users compared with a general population cohort. Pharmacoepidemiol Drug Saf. 2011;20(7):754-62.

18. Pareek A, Chandanwale AS, Oak J, Jain UK, Kapoor S. Efficacy and safety of aceclofenac in the treatment of osteoarthritis: A randomized double-blind comparative clinical trial versus diclofenac-an Indian experience. Curr Med Res Opin. 2006;22:977-88.

19. Ward DE, Veys EM, Bowdler JM, Roma J. Comparison of aceclofenac with diclofenac in the treatment of osteoarthritis. Clin Rheumatol. 1995; 14:656-62.

20. Sridhar G, Jyothirmai J, Anuradha P, Anandam B. A comparative study of efficacy and tolerability of tramadol and aceclofenac in treatment of osteoarthritis. J Evid Based Med Healthc. 20165;3:462-7.

21. Malonne H, Coffiner M, Fontaine D, Sonet B, Sereno A, Peretz A et al. Long-term tolerability of tramadol LP, a new once-daily formulation, in patients with osteoarthritis or low back pain. J Clin Pharm Ther. 2005;30:113-20.

22. Subedi M, Bajaj S, Kumar MS, Mayur YC. An overview of tramadol and its usage in pain management and future perspective. Biomed Pharmacother 2019;111:443-51.

23. Pavelka K, Peliskova Z, Stehlikova H, Ratcliffe S, Repas C. Intra individual differences in pain relief and functional improvement in osteoarthritis with diclofenac or tramadol. Clin Drug Investig. 1998;16:421-9.

24. Fowler PD, Shadforth MF, Crook PR, John VA. Plasma and synovial fluid concentrations of diclofenac sodium and its major hydroxylated metabolites during long-term treatment of rheumatoid arthritis. Eur J Clin. Pharmacol. 1983;25(3):389-94.

25. Suzuki M, Nakamura M, Mori M, Miura S, Tsuchiya M, Ishil H. Lansoprazole inhibits oxygen-derived free radical production from neutrophils activated by Helicobacter pylori. J Clin Gastroenterol. 1995;20(2):S93-6.

26. Ohara T, Arakawa T. Lansoprazole decreases peripheral blood monocytes and intercellular adhesion molecule-1-positive mononuclear cells. Dig Dis Sci. 1999;44:1710-15.

27. Roth SH. Efficacy and safety of tramadol $\mathrm{HCl}$ in breakthrough musculoskeletal pain attributed to osteoarthritis. J Rheumatol. 1998;25:1358-63.

28. Herman JH, Appel AM, Khosla RC, Hess EV. The in vitro effect of select classes of nonsteroidal antiinflammatory drugs on normal cartilage metabolism. J Rheumatol 1986;13:1014-8.

29. Park SY, Moon SH, Park MS, Oh KS, Lee HM. The effects of ketorolac injected via patient-controlled analgesia postoperatively on spinal fusion. Yonsei Med J 2005;46:245-51.

30. Jett MF, McGuirk J, Waligora D, Hunter JC. The effects of mexiletine, desipramine and fluoxetine in rat models involving central sensitization. Pain 1997;69:161-9.

31. Wassif W, Bjarnason I. A comparison of the effects of aceclofenac and diclofenac on gastrointestinal blood loss. Br J Clin Res 1992;3:109-14.

32. Accardo S, Seriolog B, Tirri G, Colombo B, Fumagali M, Lotti $G$ et al. Long-term safety profile of aceclofenac in the treatment of articular pain. Eur $\mathbf{J}$ Rheumatol Inflamm. 1995;15:35-41.

Cite this article as: Kanjiya R, Kotadia R. Comparative analysis of efficacy and tolerability of tramadol versus diclofenac in treatment of knee osteoarthritis. Int J Basic Clin Pharmacol 2021;10:1186-90. 